\section{(6) OPEN ACCESS}

\title{
AMMECR1: a single point mutation causes developmental delay, midface hypoplasia and elliptocytosis
}

\author{
Gaia Andreoletti, ${ }^{1}$ Eleanor G Seaby, ${ }^{1}$ Jennifer M Dewing, ${ }^{2}$ Ita O'Kelly, ${ }^{2}$ \\ Katherine Lachlan, ${ }^{1,3}$ Rodney D Gilbert, ${ }^{4}$ Sarah Ennis ${ }^{1}$
}

- Additional material is published online only. To view please visit the journal online (http://dx.doi.org/10.1136/ jmedgenet-2016-104100).

'Human Genetics \& Genomic Medicine, University of Southampton, Duthie Building (Mailpoint 808), Southampton General Hospital, Southampton, UK

${ }^{2}$ Centre for Human

Development, Stem Cells and Regeneration $\mathrm{HDH}$, University of Southampton, IDS Building, Southampton General Hospital Southampton, UK

${ }^{3}$ Wessex Clinical Genetics Service, University Hospital Southampton NHS Foundation Trust, Princess Anne Hospital Southampton, UK ${ }^{4}$ Wessex Regional Paediatric Nephro-Urology Service, Southampton Children's Hospital, Southampton, UK ${ }^{5}$ Faculty of Medicine, University of Southampton, Southampton, UK

Correspondence to Dr Rodney D Gilbert, University Hospital Southampton NHS Foundation Trust Honorary Senior Lecturer, University of Southampton, Southampton S016 6YD, UK; Rodney. Gilbert@uhs.nhs.uk

GA, EGS, RDG and SE contributed equally.

Received 14 June 2016 Revised 3 August 2016 Accepted 26 September 2016 Published Online First

3 November 2016

CrossMark

To cite: Andreoletti $\mathrm{G}$, Seaby EG, Dewing JM, et al. J Med Genet 2017;54:269277.

\section{ABSTRACT}

Background Deletions in the Xq22.3-Xq23 region, inclusive of $C O L 4 A 5$, have been associated with a contiguous gene deletion syndrome characterised by Alport syndrome with intellectual disability (Mental retardation), Midface hypoplasia and Elliptocytosis (AMME). The extrarenal biological and clinical significance of neighbouring genes to the Alport locus has been largely speculative. We sought to discover a genetic cause for two half-brothers presenting with nephrocalcinosis, early speech and language delay and midface hypoplasia with submucous cleft palate and bifid uvula.

Methods Whole exome sequencing was undertaken on maternal half-siblings. In-house genomic analysis included extraction of all shared variants on the $X$ chromosome in keeping with $X$-linked inheritance. Patient-specific mutants were transfected into three cell lines and microscopically visualised to assess the nuclear expression pattern of the mutant protein.

Results In the affected half-brothers, we identified a hemizygous novel non-synonymous variant of unknown significance in AMMECR1 (c.G530A; p.G177D), a gene residing in the AMME disease locus. Transfected cell lines with the p.G177D mutation showed aberrant nuclear localisation patterns when compared with the wild type. Blood films revealed the presence of elliptocytes in the older brother.

Conclusions Our study shows that a single missense mutation in AMMECR 1 causes a phenotype of midface hypoplasia, mild intellectual disability and the presence of elliptocytes, previously reported as part of a contiguous gene deletion syndrome. Functional analysis confirms mutant-specific protein dysfunction. We conclude that $A M M E C R 1$ is a critical gene in the pathogenesis of $A M M E$, causing midface hypoplasia and elliptocytosis and contributing to early speech and language delay, infantile hypotonia and hearing loss, and may play a role in dysmorphism, nephrocalcinosis and submucous cleft palate.

\section{INTRODUCTION}

The chromosomal region Xq22.3-Xq23 encompasses the entire COL4A5 gene and its adjacent genes extending towards the telomere: GUCY2F, NXT2, KCNE1L, ACSL4, TMEM164, MIR3978, AMMECR1, SNORD96B, RGAG1, TDGF3, CHRDL1, PAK3 and DCX. Deletions in this region have been associated with a contiguous gene deletion syndrome characterised by Alport syndrome with intellectual disability, midface hypoplasia and elliptocytosis (AMME (MIM: 300194)). The first report of AMME was by Jonsson et $a l^{1}$ who reported a microdeletion spanning from COL $4 A 6$ (upstream of COL4A5) to TDGF3 in two affected brothers. COL4A5, the gene underlying X-linked Alport syndrome (AS (MIM: 301050)), maps to $\mathrm{Xq} 22.3$ and is well known to cause renal failure, sensorineural hearing loss and ocular abnormalities. $^{2}$ However, mutations in COL4A5 do not account for the additional manifestations seen in AMME; this suggests that neighbouring genes of the Alport locus contribute to the rest of the phenotype. Until recently, the significance of these adjacent genes has been largely speculative.

We report on two maternal half-brothers, presenting with nephrocalcinosis and midface hypoplasia. The older sibling also has deafness and elliptocytosis. By application of next-generation sequencing, we identified a novel point mutation in AMMECR1 (c.G530A; p.G177D) present in the half-siblings and carried in the mother. We show that this variant alone causes midface hypoplasia, speech and language delay and elliptocytosis, emphasising the critical importance of AMMECR1 in the pathogenesis of AMME.

\section{METHODS}

Two maternal Caucasian half-siblings presenting with overlapping phenotypes (figure 1) were ascertained through the paediatric nephrology service at University Hospital Southampton.

\section{Proband II(1)}

Following a pregnancy complicated by extreme hyperemesis, proband II(1) was delivered by elective caesarean section for breech presentation weighing $3.59 \mathrm{~kg}$ (just above 50th centile). Antenata screening showed evidence of fetal nuchal oedema, bilateral talipes, short femurs and decreased liquor. An amniocentesis showed a normal 46,XY karyotype. Shortly after birth, proband II(1) was noted to have hypotonia and poor feeding. Methylation testing of SNRPN exon 1 in the PWS/AS critical region was normal. Apparently short femora, bilateral talipes, widely spaced nipples, a flat facial profile with a flat nasal bridge, downslanting palpebral fissures, thin upper lip, micrognathia, short neck, folded upper helix to the ears, fifth finger clinodactyly and additional right nipple were noted. He had surgical correction for a submucous 
Figure 1 (A) Family pedigree for proband II(1), and maternal half-brother II(2) with genotype information for all individuals tested. Affected individuals are in black. The maternal parents were phenotypically normal. Asterisk defines individuals who underwent whole exome sequencing. (B) Photographs of proband II(1) (taken at 11 months) (left) and proband II(2) (taken at 2 years and 5 months) (right).
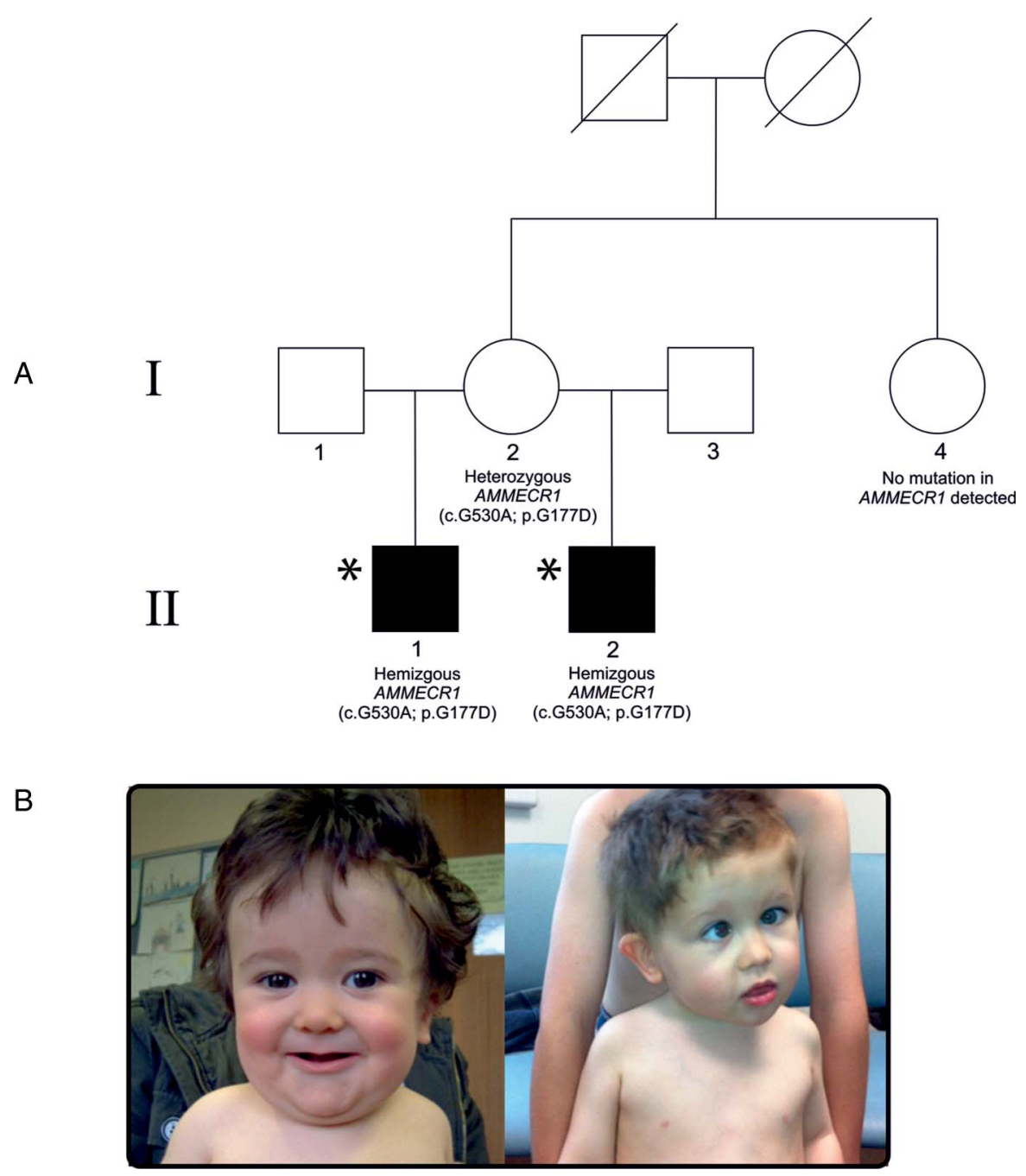

cleft palate and bifid uvula. An abdominal ultrasound scan showed possible nephrocalcinosis. An ultrasound of the head was normal.

Aged 2 months, he underwent bilateral percutaneous achilles tenotomies. His length was just above the 0.4 th centile, weight on the 2 nd centile and with a disproportionately large head circumference on the 50th centile. Bilateral nephrocalcinosis was confirmed on a repeat ultrasound scan. At seven and a half months, following application of the Alberta Infant Motor Scale, ${ }^{3}$ he was assessed to have mild-to-moderate delay in motor and communication skills. Diagnoses of Stickler syndrome and chondrodysplasia punctata were considered. Very long chain fatty acid tests were normal. Vision and fundal examination were normal on formal ophthalmic examination. Skeletal surveys performed just before his first birthday, and repeated when aged 4, reported no skeletal abnormality and specifically no epiphysial stippling. He was discharged from physiotherapy, occupational therapy and speech and language therapy before his second birthday, having made improvements in his gross motor development and feeding following cleft palate repair. Aged 23 months, he had a normal brain MRI with no evidence of hypothalamic or pituitary abnormality, ruling out a midline defect. He did not get his first teeth until he was 21 months old.

Aged 3, his short stature (0.4th centile) was calculated to be within the target centile range for his parents' heights. His head remained disproportionately large (50th centile) with weight on the 2 nd centile. Aged 4, he was noted to have mild facial dysmorphology consisting of a flat midface with a large, broad forehead and small mouth with crowded teeth. There were bulbous tips to his toes and marked lumbar lordosis without scoliosis. There was a normal sitting height to leg length ratio. He had started mainstream school; however, there was still parental concern regarding slow progress and poor concentration. $\mathrm{He}$ had repeatedly elevated urine calcium:creatinine ratios, but a persistently normal plasma calcium concentration. Further ultrasound scans corroborated previous findings of nephrocalcinosis. At his most recent review, aged 11, proband II(1) remained in mainstream school. His facial dysmorphic features were much less apparent than observed previously, and his growth parameters were unchanged. He had bilateral mixed hearing loss requiring hearing aids; sensorineural hearing loss was first detected at age 3 , despite a normal audiogram 1 year previously. His bone conduction threshold was $35-45 \mathrm{~dB}$ bilaterally, whereas air conduction showed a threshold of $45-50 \mathrm{~dB}$ bilaterally. He had also begun to suffer from migraines. He had mild joint hypermobility but much improved hypotonia. He had persistent nephrocalcinosis with intermittent hypercalciuria.

\section{Younger maternal half-brother II(2)}

Proband II(2) was born by emergency caesarean section for failure to progress at 39 weeks' gestation weighing $3.40 \mathrm{~kg}$. His pregnancy was complicated by polyhydramnios and hyperemesis 
similar to his older half-brother. Otherwise, antenatal screening was normal. He had congenital dysplasia of the hips treated with a Pavlik harness. Retrospective review of early radiographs showed no evidence of epiphysial stippling. Shortly after birth, it was noted that he shared dysmorphic facial features with his half-brother. He also had a submucous cleft palate, bifid uvula and short stature. Aged 1, he underwent a renal ultrasound scan, which revealed bilateral renal dysplasia. The right kidney measured $5.4 \mathrm{~cm}$ and the left was $6 \mathrm{~cm}$. There was early nephrocalcinosis. A simple cyst was seen in the midpole of the left kidney. He did not have the significant infantile hypotonia observed in his brother and met his motor milestones appropriately. However, he had more significant speech and language delay, with no words at 2 years of age. Aged 3, he had made progress and was putting two words together. Similar to his brother, his growth parameters followed below the 0.4th centile for height, with weight between the 2nd and 9th centile and head circumference between the 25th and 50th centiles. He had joint hypermobility, right esotropia and viral-induced wheeze. Aged 4, an audiogram showed air conduction thresholds of $20 \mathrm{~dB}$ bilaterally, just within the normal range. Bone conduction was tested only on the right with a threshold of $10 \mathrm{~dB}$ across the frequency range. This audiogram showed deterioration from one previously. Aged 5, proband II(2) was developing well and displayed a degree of developmental catch up with improved speech and language skills; he attended a normal school. His facial dysmorphology was also less apparent.

\section{Mother I(2)}

Their mother was of normal intelligence. Renal ultrasound scan performed when she was 34 years was normal. She had normal renal function and there was no evidence of hypercalciuria. The maternal grandparents were deceased but were reported as showing none of the abnormal phenotypic features of the probands. There were no other male relatives.

\section{Clinical genetic testing}

An array comparative genomic hybridisation (CGH) was performed on proband II(1) and showed no copy number variations (CNVs; Agilent $44 \mathrm{~K}$ oligo array-CGH). A multidisciplinary discussion informed selection of a gene candidate, OCRL1, an X-linked gene involved in Lowe oculocerebrorenal syndrome, Fanconi syndrome, intellectual disability and nephrocalcinosis (MIM: 309000). ${ }^{4}$ Sanger sequencing of OCRL1 revealed no mutations in either sibling. As a result, the half-siblings were referred to the Southampton Genomic Informatics Group for whole exome sequencing.

\section{DNA extraction}

Genomic DNA (gDNA) was extracted from peripheral venous blood samples collected in an ethylenediaminetetraacetic acid tube. DNA concentration was estimated using the Qubit 2.0 Fluorometer. The average gDNA yield obtained was $150 \mu \mathrm{g} /$ $\mathrm{mL}$. Approximately, $20 \mu \mathrm{g}$ of gDNA was used per patient for next-generation sequencing.

\section{Whole exome sequencing data generation and data analysis}

Whole exome sequencing was performed on two maternal halfsiblings using the Agilent SureSelect Human all Exon $51 \mathrm{Mb}$ V.5 capture kit. As previously described, ${ }^{5}{ }^{6}$ FASTQ raw data generated from Illumina paired-end sequencing were aligned against the human reference genome (hg19) using Novoalign (novoalign/2.08.02). SAMtools mpileup tool (samtools $/ 0.1 .19)^{7}$ was used to detect the variation from the mapping information to call SNPs and short INDELs from the alignment file. ANNOVAR (annovar/21 February 2013) ${ }^{8}$ was applied for variant annotation against a database of RefSeq transcripts. A bespoke script was used to assign individual variants as: 'novel' if they were not previously reported in the dbSNP137 databases, ${ }^{9} 1000$ Genomes Project, ${ }^{10}$ the Exome Variant Server (EVS) of European Americans of the NHLI-ESP project with 6500 exomes (http://evs.gs.washington.edu/EVS/), ${ }^{11}$ in 46 unrelated human subjects sequenced by Complete Genomics ${ }^{12}$ or in the Southampton database of reference exomes. Resultant variant files for each individual were subjected to further in-house quality control tests to detect DNA sample contamination and ensure sex concordance by assessing autosomal and $\mathrm{X}$ chromosome heterozygosity. ${ }^{13}$ Variant sharing between the two siblings was assessed to confirm sample relationships, and VerifyBamID ${ }^{14}$ was used to check for sample contamination. Sample provenance was confirmed by independent genotyping of a validated SNP panel, developed specifically for exome data. ${ }^{15} \mathrm{CNVs}$ across the Xq22.3-Xq23 region were assessed using the R package ExomeDepth. ${ }^{16}$

\section{Gene selection}

Despite no mutations identified by Sanger sequencing, OCRL1 was reassessed using an alternative sequencing resource. The segregation pattern in the family was strongly suggestive of $\mathrm{X}$-linked recessive inheritance; both maternal half-siblings were affected despite a healthy mother, which suggested they were hemizygous for the mutant allele. The genomic analysis was, therefore, extended to extract all variations on the $\mathrm{X}$ chromosome common to the half-siblings. Additionally, we screened for rare, damaging variants across $\mathrm{Xq} 22.3-\mathrm{Xq} 23$ as well as a panel of known genes associated with idiopathic hypercalciuria/ nephrocalcinosis (see online supplementary methods).

\section{Site-directed mutagenesis}

PCMV6-AC-GFP-tagged AMMECR1 vector was obtained from Origene (RG212378). Site-directed mutagenesis was carried out to achieve the G>A point mutation within position 177 in exon 2 of variant 1 of AMMECR1 to confer substitution of amino acid glycine with aspartate. Primers were designed containing the G>A point mutation: forward primer: CGTGGATGCAT AGATACTTTTTCTGCC; reverse primer: GGCAGAAAAAGT ATCTATGCATCCACG. PCR mutagenesis was carried out using Pfu Ultra DNA polymerase (Agilent Technologies, Stockport, UK) and the following thermo cycle: $2 \mathrm{~min}$ at $95^{\circ} \mathrm{C},(12 \times$ cycles of $1 \mathrm{~min}$ at $95^{\circ} \mathrm{C}, 1 \mathrm{~min}$ at $55^{\circ} \mathrm{C}, 7.5 \mathrm{~min}$ at $68^{\circ} \mathrm{C}$ ), 10 min at $68^{\circ} \mathrm{C} .{ }^{17}$ DNA was digested with Dpn1 for 1 hour at $37^{\circ} \mathrm{C}$ to cleave the parental DNA template. The mutated vector was sequenced to confirm the AMMECR1 mutation (Source BioScience).

\section{Cell culture}

HEK293, COS-7 and HeLa cells were plated on $22 \mathrm{~mm}$ sterile coverslips in six-well plates in dulbecco modified eagle medium (DMEM) $/ 10 \%$ fetal calf serum (FCS). Cells in each well were transiently transfected with $3 \mu \mathrm{g}$ of either wild-type or mutant GFP-tagged AMMECR1 using jetPEI transfection reagent, according to the supplier's instructions (Polyplus, Source BioScience, Nottingham, UK). Cells transfected with jetPEI reagent in the absence of DNA were used as a negative control. DNA-PEI complexes were removed from cells after 4 hours and replaced with fresh DMEM/10\% FCS/1\% Pen/strep. 


\section{Microscopy}

Twenty-four hours after transient transfection, cells were fixed with $4 \%(\mathrm{w} / \mathrm{v})$ formaldehyde in phosphate buffered saline (PBS) for $7 \mathrm{~min}$ at room temperature and mounted in VectaShield mounting medium containing the nuclear stain, 4',6-diamidino2-phenylindole (DAPI) (VectorLabs). Coverslips were mounted and visualised using a fluorescent microscope (Zeiss).

\section{RESULTS}

\section{Quality control analysis}

Exome data were high quality, evidenced by $80 \%$ mapped coverage at a read depth of $>20$. For both samples, the average depth of coverage was $\geq 60$, online supplementary table 1 . The two samples from the affected siblings selected for exome analysis exhibited expected variant sharing for second-degree relatives and no excess of sharing with any other sample on the same despatch DNA plate. Autosomal and X-chromosome heterozygosity were consistent with gender. VerifyBamID ${ }^{14}$ did not indicate any presence of contamination, and the application of a SNP tracking panel ${ }^{15}$ confirmed sample provenance.

\section{Targeted gene analysis}

No coding variation was found in OCRL1 consistent with previous Sanger sequencing results. Therefore, the analysis was extended to include all coding mutations across the $\mathrm{X}$ chromosomes of the two brothers and three novel nonsynonymous mutations satisfied the filtering criteria (as outlined in table 1): AMMECR1 (c.G530A; p.G177D), KDM5C (c.4082A; p.R1361K) and MAGEC1 (c.G919A; p.V307M). The MAGEC1 (p.V307M) variant was poorly conserved (PhyloP ${ }^{18}$ score of 0.868789 ) and was not predicted to be damaging and thus removed from downstream analysis. Although KDM5C has been associated with Claes-Jensen Type Intellectual Disability (MIM: 314690), which is a syndrome that has been characterised by severe mental retardation, microcephaly and large feet, we removed the novel variant (p.R1361K) from the downstream analysis because it was not predicted to be damaging by the in silico tools CADD ${ }^{19}$ and Polyphen $2^{20}$ (score of 1.2 and 0.033 , respectively) Furthermore, KDM5C has been associated with mental retardation, whereas we describe a mild developmental delay inconsistent with the severity and nature of the phenotype of Claes-Jensen. The novel non-synonymous variant of unknown significance in AMMECR1 (c.G530A; p. G177D) warranted further scrutiny with high prediction scores of pathogenicity (Phylop, Polyphen2, CADD and GERP++ scored this variant $0.998909,1,5.5$ and 5.27, respectively). Moreover, AMMECR1 has previously been deleted in patients with Alport syndrome, mental retardation, midface hypoplasia and elliptocytosis. ${ }^{1} 2122$ No rare variants were identified in known hypercalciuria genes (see online supplementary results
1), although CLDN14 and PTH had suboptimal gene coverage by the Agilent V5 capture kit (45\% and $86 \%$, respectively). All other hypercalciuria genes had $>90 \%$ coverage.

\section{Mutation within AMMECR1}

The AMMECR1 mutation segregating in our family causes a $\mathrm{G}>\mathrm{A}$ change at genomic position 530, leading to a glycine to aspartate change at codon 177 in exon 2. AMMECR1 encodes a protein with a nuclear location whose function is still unknown. The C-terminal region of AMMECR1 (from amino acid residue 122 to 333) is well conserved, and homologues appear in species ranging from bacteria and archaea to eukaryotes. Exon 2 of AMMECR1 encodes a domain consisting of six amino acids identically conserved throughout the course of evolution. The putative nuclear localisation and the presence of a glycine-rich $\mathrm{N}$ terminus raise the possibility that $A M M E C R 1$ has a regulatory role.

No CNVs or rare variants were observed in either of the affected brothers within the Xq22.3-Xq.23 region (average read depth of 49.66). We confirmed the AMMECR1 mutation using traditional Sanger techniques and demonstrated hemizygous status in the two half-brothers and heterozygous carrier status in the unaffected mother. The probands' maternal aunt did not carry the AMMECR1 mutation.

\section{Elliptocytosis}

With evidence to support an association between AMMECR1 and elliptocytosis, blood films were requested on the brothers at ages 10 and 4, and both smears were reported blindly. The younger brother, proband II(2), had a normal blood film with no evidence of elliptocytosis, but the older brother, proband II (1), had an abnormal film with scattered elliptocytes and anisocytosis. A repeat blood film on proband II(1) 10 months later confirmed the presence of elliptocytes, though the findings were subtle.

\section{Functional analysis}

Analysis of AMMECR1 protein was assessed by GFP expression in transfected cell lines. Colocalisation of GFP with the nuclear DAPI stain was observed in both mutant and wild-type transfected cells, showing localisation of AMMECR1 protein within the nucleus (figure 2). The expression pattern of wild-type AMMECR1 was spherical, overlaying the DAPI staining completely. Mutant AMMECR1 expression was distinct from wild type in all three cell lines tested, with HEK293, COS-7 and HeLa cells exhibiting non-uniform GFP expression within the nucleus. The number of GFP-positive cells was also reduced following transfection with mutant AMMECR1 compared with the wild type. These data confirm that the $\mathrm{G}>\mathrm{A}$ point mutation

Table 1 Three novel non-synonymous mutations that satisfied the filtering criteria across the 323 variants shared on the $X$ chromosomes between the two half-brothers

\begin{tabular}{|c|c|c|c|c|c|c|c|c|}
\hline Chromosome & $\begin{array}{l}\text { bp } \\
\text { position } \\
\text { (hg19) }\end{array}$ & Gene & $\begin{array}{l}\text { Variant } \\
\text { type }\end{array}$ & Variant info & Phylop $^{+}$ & Polyphen & CADD & Gerp $^{++}$ \\
\hline $\mathrm{x}$ & 109507771 & AMMECR1 & ns & AMMECR1:NM_015365:exon2:c.G530A:p.G177D & 0.998909 & 1 & 5.776826 & 5.27 \\
\hline$x$ & 53221984 & KDM5C & ns & KDM5C:NM_001146702:exon24:c.G4082A:p.R1361K & & 0.033 & 1.26541 & \\
\hline $\mathrm{x}$ & 140994109 & MAGEC1 & ns & MAGEC1:NM_005462:exon4:c.G919A:p.V307M & 0.868789 & 0.512688 & & 0.157 \\
\hline
\end{tabular}

A total of 18049 variants were shared between the half-siblings. The filtering strategy applied was limited to shared variants on the $X$ chromosome only ( 323 annotated). 318 variants were removed due to their occurrence within the Southampton control cohort of exomes $(n=156)$ regardless of annotated zygosity. Of the five remaining variants, one was removed due to its synonymous annotation, and another was disregarded due to its low MaxEnt splicing score $(<3)$. Three novel (absent from ExAC server, dbSNP and the Southampton inhouse control database) non-synonymous variants satisfied the full filtering criteria. Ns, non-synonymous; sp, splicing. 


\section{WILD TYPE}
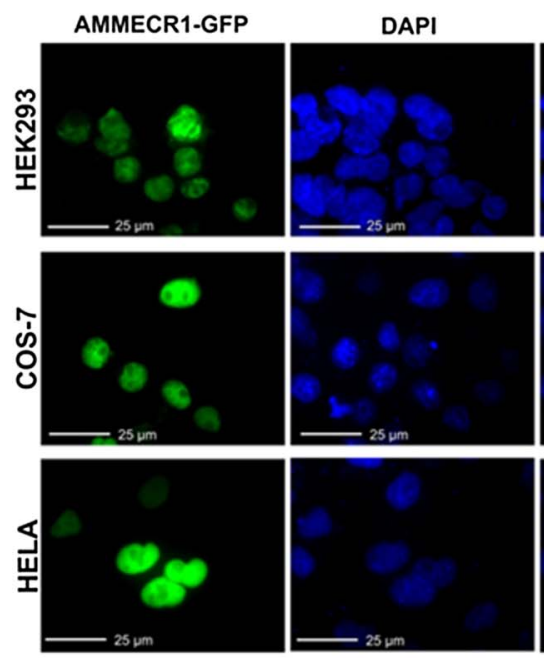
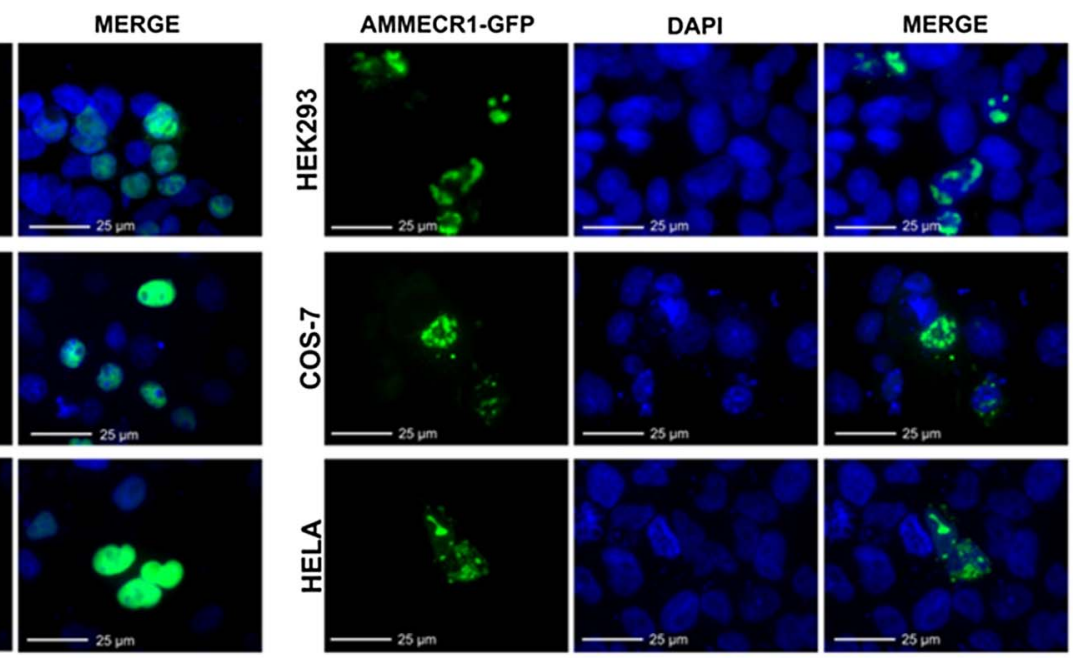

Figure 2 G>A point mutation within position 177 of AMMECR1 gene alters the nuclear expression pattern of the protein. Representative images of HEK293, COS-7 and HeLa cells 24 hours after transfection with a vector containing wild-type or mutant GFP-tagged AMMECR1. Nuclear localisation of AMMECR1 was observed in both wild type and mutant; however, the non-uniform expression pattern of AMMECR1 was only found in cells transfected with mutant AMMECR1.

within position 177 of exon 2 of $A M M E C R 1$ affects the expression pattern of this protein within the nucleus.

\section{DISCUSSION}

We have identified a single base substitution $(G>A)$ causing a missense mutation in which a non-polar glycine amino acid residue is replaced with a negatively charged aspartate in the AMMECR1 protein. The observed variant codes for a highly conserved residue of AMMECR1 within a highly conserved six-amino-acid motif (LRGCIG). The variant appears novel to this pedigree not occurring in our database of local exomes nor in the publicly available variant repositories.

Functional analysis shows that the $\mathrm{G}>\mathrm{A}$ point mutation within amino acid 177 of the AMMECR1 protein affects nuclear localisation. Proteasome complexes found within the cytoplasm and nucleus are responsible for targeted degradation of mal-folded or damaged proteins occurring due to missense or non-sense mutations, as well as denaturation and biosynthetic errors. $^{23} 24$ The expression pattern of mutated AMMECR1 we observed within the nucleus is consistent with the altered protein being targeted for degradation by this system. The localisation pattern of the mutant AMMECR1-GFP protein has similarity with the localisation of subunits of the $20 \mathrm{~S}$ nuclear proteasome, previously described by Baldin et $a .^{25}$ The mutated AMMECR1 protein may therefore be targeted for degradation within the nucleus. Degradation of AMMECR1 is also consistent with reduced GFP expression observed in cells transfected with mutant AMMECR1 compared with wild type. Disease states caused by proteolytic degradation of mal-folded proteins as a result of missense mutations include cystic fibrosis, where targeted degradation of the mutant form of the cystic fibrosis transmembrane conductance regulator (CFTR) protein, caused by a missense mutation, prevents normal functioning. ${ }^{24}$

Literature describing AMMECR1 is sparse; its description and name (Alport syndrome, Mental retardation, Midface hypoplasia and Elliptocytosis Chromosomal Region gene 1) is derived from its cytogenic location within Xq22.3-Xq23 and its previous association with a very rare contiguous gene deletion syndrome originally named Alport syndrome, mental retardation, midface hypoplasia and elliptocytosis (AMME). Jonsson et $a l^{1}$ first described AMME caused by a deletion including COL $4 A 5$ (Xq22.3) extending proximally to include AMMECR1.

We found a striking resemblance between proband II(1) harbouring a single point mutation in AMMECR1 and the patient originally reported by Jonsson et al (figure 3). Contiguous gene deletions of varying sizes within Xq22.3-Xq23 manifest in phenotypes that partially or completely overlap the AMME disease spectrum have been described in five previous reports of which three were inclusive of the AMMECR1 gene (figure 4). Most of these deletions involve COL $4 A 5$, the major cause of Alport syndrome, yet mutations in COL $4 A 5$ do not account for the haematological, dysmorphic and developmental characteristics described in AMME. Therefore, it was surmised that these extra-renal abnormalities were attributable to disruption of genes adjacent to $C O L 4 A 5$, describing a new contiguous gene deletion syndrome. Subsequently, there has been speculation regarding the roles of genes in this region; however, for the most part their biological significance has been unresolved. Although our report is based on a single family and we must be tentative in drawing firm conclusions without further supportive evidence, the variant is novel. That said, mutant-specific functional evidence and the strong phenotypic overlap between the dysmorphology observed in this study and the original AMME case by Jonsson et al advance our understanding of the phenotypic/genotypic overlap between a single point mutation in AMMECR1 and this contiguous gene deletion syndrome.

Although the single point mutation we observe has clear overlapping features of AMME with previously published cases, this is in the absence of any Alport syndrome. The renal manifestations we describe are clinically disparate from Alport syndrome, consistent with an absence of any COL4A5 mutations. However, we are mindful that exome data cannot resolve (not uncommon) deep intronic variants in COL4A5, and this is a limitation of our study. As expected, haematuria is only observed in Xq22.3-Xq23 regional deletions that overlap COL4A5. Contrastingly, sensorineural hearing loss is consistently observed across all case studies, regardless of direct COL4A5 involvement. Proband II(1) in our study has hearing 

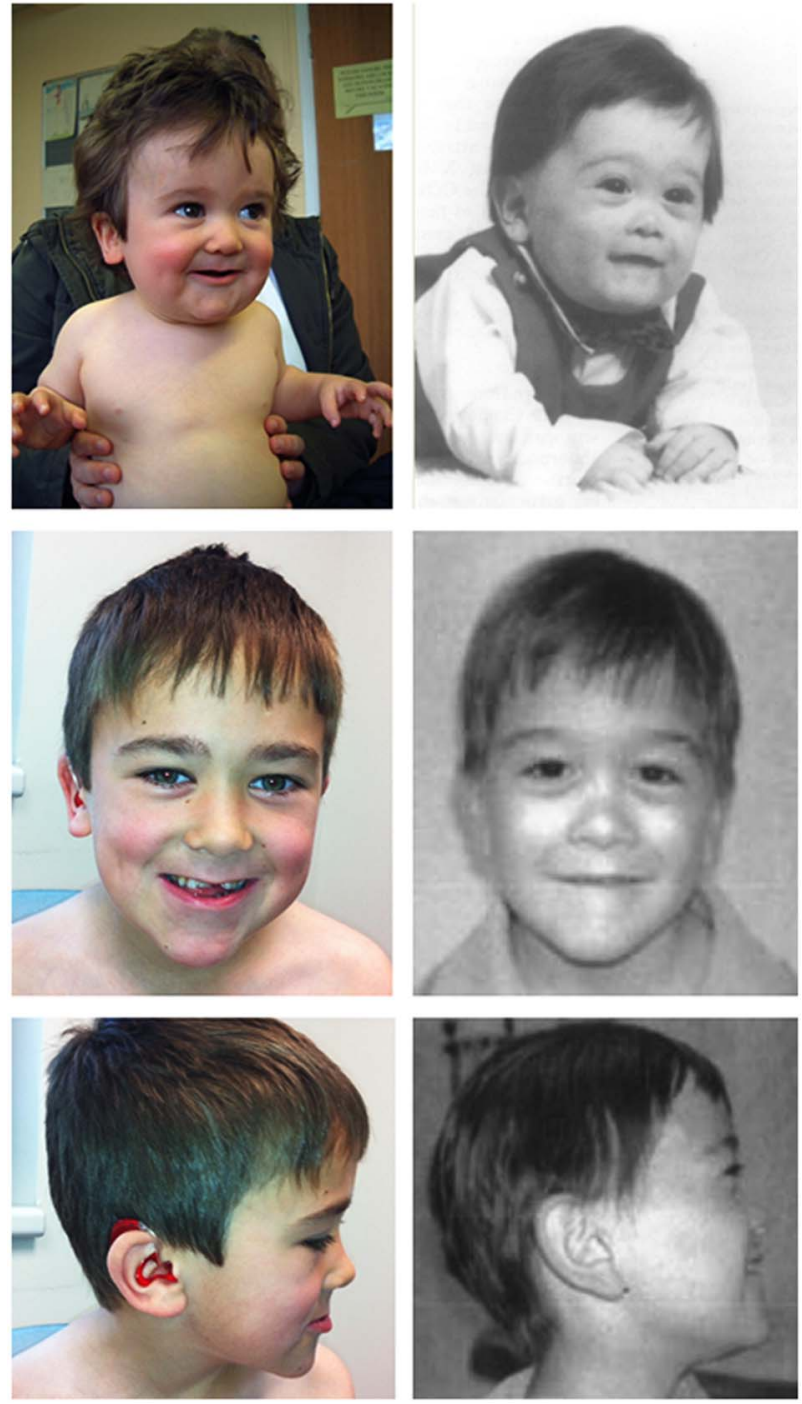

Figure 3 Colour photographs of proband II(1) (left) taken at 11 months and 9 years compared with black and white photos (right) of the patient described by Jonsson et al with features of X-linked AMME, taken at 6 months and 12 years. The facial features shared between our study and that of Jonsson et al are remarkably striking; shared facial dysmorphology includes a hypoplastic and flat midface, short neck, thin upper lip and a small jaw.

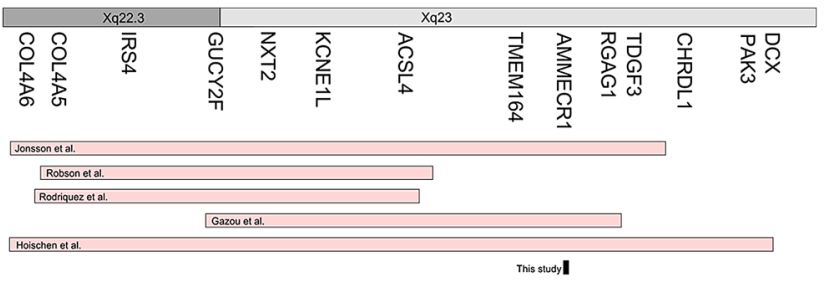

Figure 4 A comparison of deletions across Xq22.3-23 (pink) that have been associated with AMME in five separate reports, in addition to the point mutation in AMMECR1 from this study (black).

loss, as does the proband reported by Gazou et $a l^{26}$ who harboured a deletion not involving COL $4 A 5$ but inclusive of the AMMECR1 gene. This may suggest that AMMECR1 is pathogenically implicated in hearing loss, either alone, or as a modifier gene to COL4A5 due to their direct protein-protein interaction (see online supplementary figure 1). The sensorineural hearing loss in proband II(1) presented at age 3, earlier than is typical in X-linked Alport syndrome and subsequently progressed. His half-brother, at aged 4 , has air conduction values on the cusp of abnormality. His audiogram shows deterioration from one previously, suggesting a progressive pattern of hearing loss without congenital onset.

Intellectual disability is prevalent among individuals with deletions across the $\mathrm{Xq} 22.3-23$ region; however, the degree is varied. A critical region was identified by Meloni et al, ${ }^{27}$ who identified four genes of interest (ACSL4, KCNE1L, NXT2 and GUCY2F). ACSL4 has since been implicated in non-specific $\mathrm{X}$-linked mental retardation (MIM: 300387), favouring this gene as the cause of intellectual disability in Xq22.3-Xq23 deletions. ${ }^{26}$ ACSL 4 is deleted in all the microdeletion reports (figure 4). Gazou et al have stated that "Deletion of ACSL4 is probably the major, and possibly only, causative factor for intellectual disability (ID)".... The moderate degree of ID is similar to patients with point mutations in ACSL4 and does therefore not suggest additive effects by other genes. Absent or delayed speech is the predominant phenotype in individuals reported by Rodriguez et $a l,{ }^{22}$ Robson et $a l^{28}$ and Jonsson et $a l^{1}$ but this was of a greater severity than reported in these half-siblings (table 2). Standardised, quantifiable measures would aid in accurately mapping intellectual disability severity to chromosomal regions. We observed delayed speech, with no concerns by the time the children attended school, although they did have delayed reading and writing ability and there was parental concern regarding slow progress and concentration difficulties. Of course, delayed speech may be attributable to hearing abnormalities or the submucous cleft palates. Both abnormalities were corrected (with hearing aids and cleft palate repair), which may explain the improvement in speech and language development. Other reports ${ }^{1} 22 \quad 2628$ include generalised hypotonia, contributing to developmental delay. Proband II(1) had significant infantile hypotonia ${ }^{1}$ and delay in gross motor skills in addition. This may be in part secondary to joint hypermobility, which persisted through childhood. As AMMECR1 is associated in the published literature with ACSL4 ${ }^{29}$ (see online supplementary figure 1), we propose that this may explain the speech and language delay and early motor delay observed.

Midface hypoplasia is present in all but one (Gazou et al) case studies involving the AMME locus. ${ }^{26}$ While no other reports include cleft palate, midface hypoplasia is often observed in individuals with this congenital abnormality. The midface hypoplasia may represent a minimal expression of this phenotype. The dysmorphology reported is variable and includes anteverted nares, downslanting palpebral fissures, peripalpebral fullness, fetal pads, deep-set eyes, wide nasal bridge, broad nasal tip, long philtrum, thin upper lip, downturned mouth, widely spaced teeth, supernumery teeth, facial hypotonia, second toe syndactyly and clinodactyly. Of note, as with our study, there are reports that the facial dysmorphism lessens in severity over time. ${ }^{21}$

Within the AMME locus, elliptocytosis only occurs in regions inclusive of AMMECR1, although its penetrance is varied; elliptocytes were observed only in the blood films of the elder brother at age 10 and not in the younger sibling at age 4 . Haematological abnormalities were also absent from the probands reported by Gazou et $a l^{26}$ and Hoischen $e a^{30}$ (although the Hoischen proband was female with a uniallelic deletion). It is noteworthy, however, that the elliptocytosis is a mild phenotype (figure 5). Scattered elliptocytes were present and reported on both blood films; however, their subtlety may not warrant an obvious elliptocytosis diagnosis; that said, we report a similar 


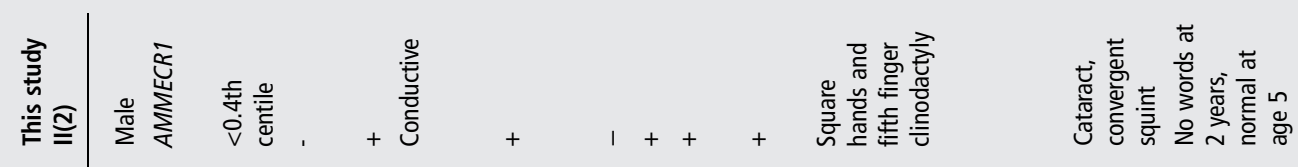

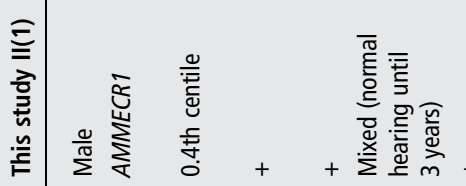

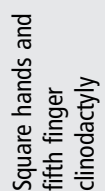

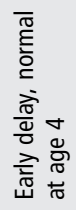

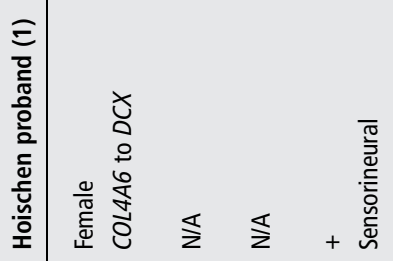

咅

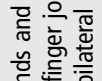

空

离

产

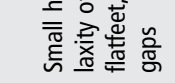

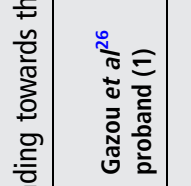

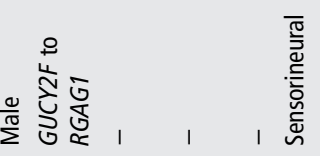

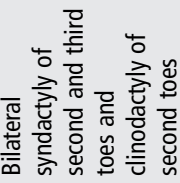

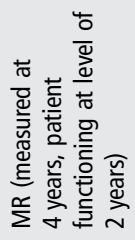

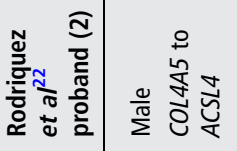

तั่

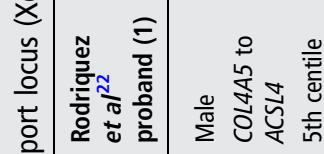

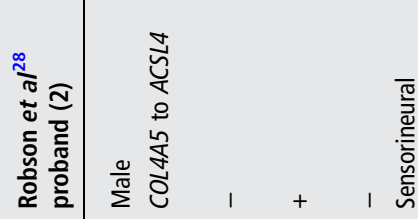

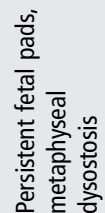

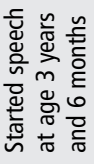

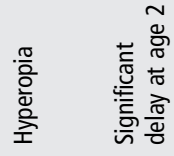

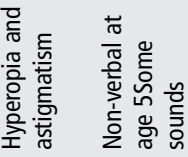

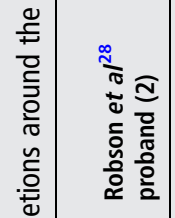

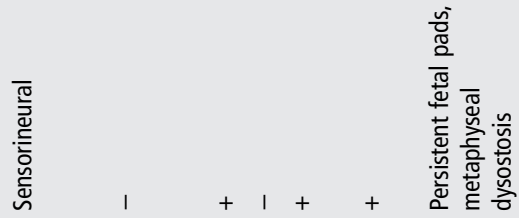

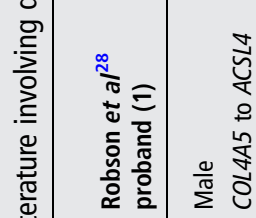

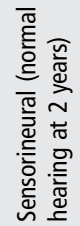

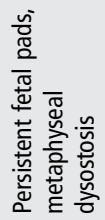

$\frac{\ltimes}{\Sigma}$

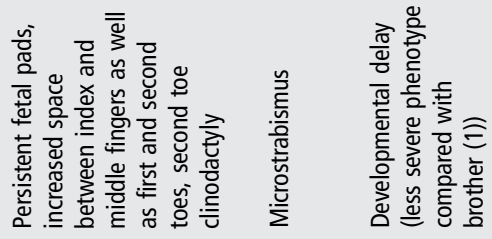

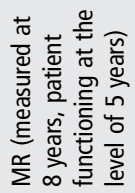

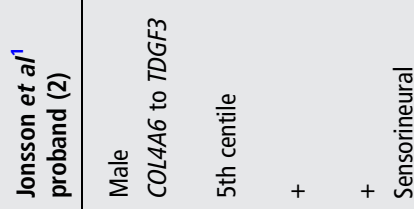

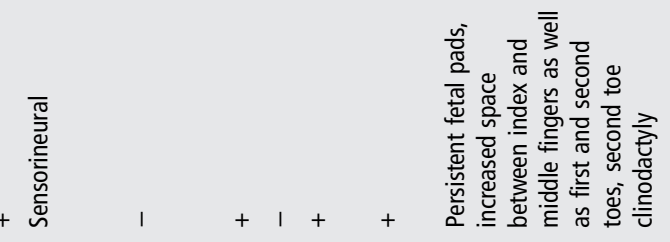

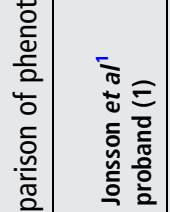

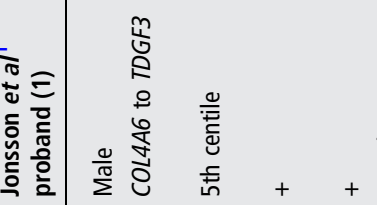

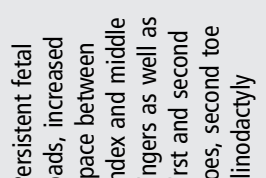

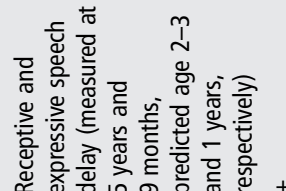

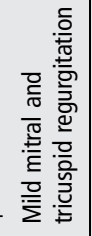




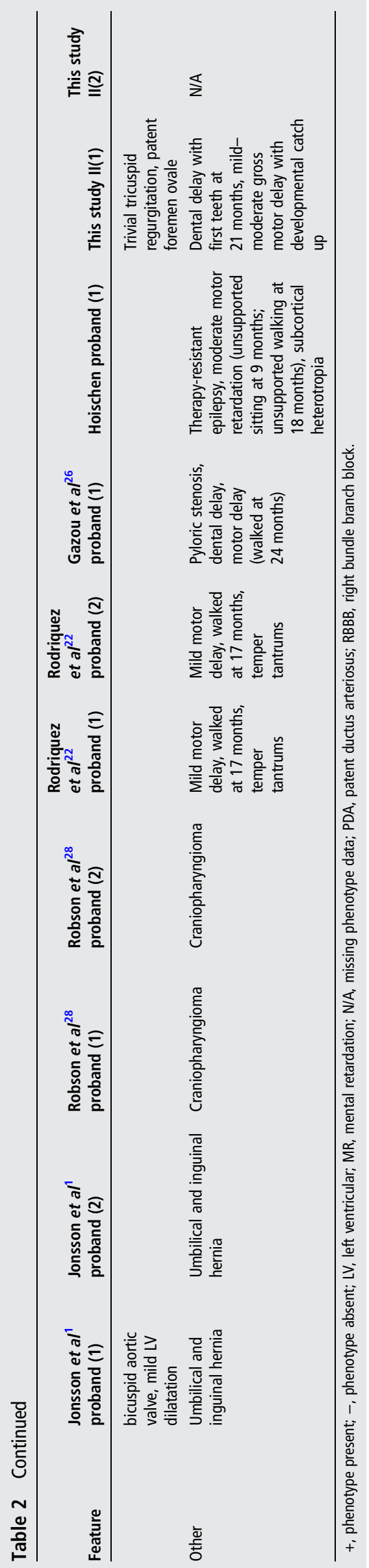

scattering of elliptotic cells consistent with the (mild to moderate) 'elliptocytosis' originally reported by Jonsson et al. ${ }^{1}$ Absence of elliptocytosis in other studies with microdeletions spanning AMMECR1 may be due to laboratory under-reporting, particularly if the red cell morphology is subtle. Moreover, aberrant red cell morphology was absent in the younger half-sibling from this study, which may instead reflect variable penetrance or demonstrate a delay in phenotype onset; however, elliptocytosis was detected at 15 months in one of the affected brothers from the Jonsson et al study, where the entire AMMECR1 gene was deleted.

The single point mutation in AMMECR1 in this study accounts for early speech and language delay, hypotonia, midface hypoplasia and elliptocytosis, and is found in association with nephrocalcinosis, cleft palate, bifid uvula, hearing loss, hypercalciuria, strabismus, cataracts, hypermobility, hypotonia and delay in eruption of primary dentition; although the phenotypic penetrance is varied. The strong interactions between AMMECR1 and neighbouring proteins of the AMME locus render genotype/phenotype stratification difficult. Nephrocalcinosis, hypercalciuria, cataracts (in proband II(2)), submucous cleft palate and bifid uvula are unique to our study and may be entirely incidental; we do not have sufficient evidence in support of any causal relationship between AMMECR1 and these manifestations. It is possible, though unlikely, that the half-brothers may have a second (unrelated) condition that explains their additional features. We excluded known genetic causes for nephrocalcinosis and idiopathic hypercalciuria in 12

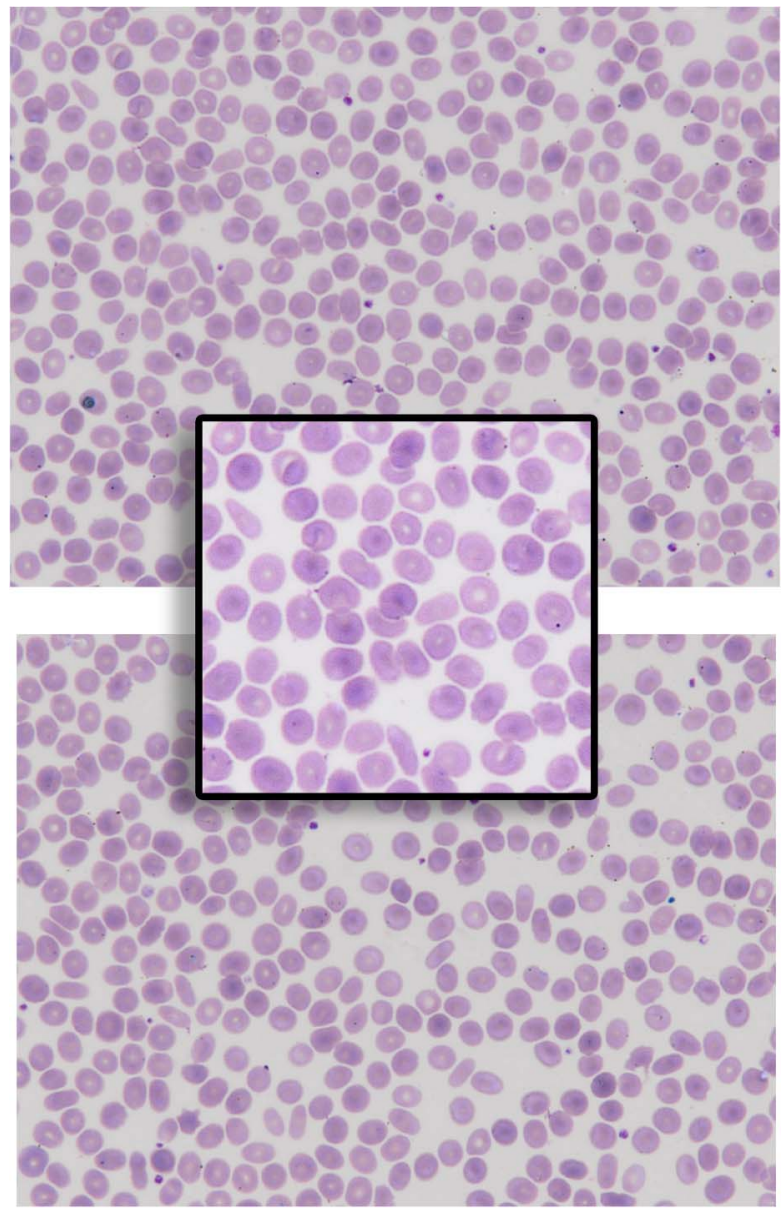

Figure 5 A peripheral blood film (two fields of view and one closer image) of proband II(1) reported as 'elliptical cells seen'. 
of 14 genes assessed. Two genes, PTH and CLDN14, had suboptimal gene coverage; and although no variants were found, we are cautious to exclude them. However, we are reassured that both CLDN14 and PTH are not on the X chromosome.

\section{CONCLUSIONS}

In this study, we have applied contemporary sequencing technology and unbiased filtering to a half-brother pair and identified a single point mutation within an X-linked gene that causes a severe phenotype, previously described as part of a contiguous gene deletion syndrome. Functional analysis shows that the novel mutation in AMMECR1 affects protein localisation, possibly due to degradation of the mal-folded protein. We propose that AMMECR1 has a critical role in the extrarenal manifestations of AMME, either alone or due to its predicted proteinprotein interactions with other genes in the AMME locus. Our study narrows the AMME locus to a single point mutation in AMMECR1 that appears critical in the pathogenesis of midface hypoplasia and elliptocytosis, and contributes to early speech and language delay, hypotonia and hearing loss, and may, in addition, play a role in dysmorphism, nephrocalcinosis and submucous cleft palate.

Acknowledgements The authors are grateful to the participating family. Gratitude extends to Nikki Graham for all her laboratory expertise in extracting and storing DNA prior to whole exome sequencing, and Drs Mary Morgan and Frank Boulton for their haematological expertise.

Contributors GA: Exome analysis, co-wrote the first draft of the manuscript, and revised and approved final version. EGS: Exome analysis, collated phenotypic information, co-wrote the first draft, revised manuscript and approved the final version. JMD and $\mathrm{IO}^{\prime} \mathrm{K}$ performed transfection experiments, wrote the relevant part of the manuscript and approved the final version. KL: Clinical genetic input including phenotypic information. Revised manuscript and approved final version. RDG: Conceived project jointly with SE. Provided phenotypic data, revised manuscript and approved the final version. SE: Conceived project jointly with RDG. Supervised exomic analysis. Revised manuscript and approved the final version.

Funding This work was financially supported by the Biotechnology and Biological Sciences Research council (BBSRC), grant number BB/J008168/1. Miss Andreoletti is supported by The Crohn's in Childhood Research Association (CIRCA) and The Gerald Kerkut Charitable Trust.

Competing interests None declared.

Provenance and peer review Not commissioned; externally peer reviewed.

Data sharing statement No additional, unpublished data are included in this study.

Open Access This is an Open Access article distributed in accordance with the terms of the Creative Commons Attribution (CC BY 4.0) license, which permits others to distribute, remix, adapt and build upon this work, for commercial use, provided the original work is properly cited. See: http://creativecommons.org/licenses/ by/4.0/

\section{REFERENCES}

1 Jonsson JJ, Renieri A, Gallagher PG, Kashtan CE, Cherniske EM, Bruttini M, Piccini M, Vitelli F, Ballabio A, Pober B. Alport syndrome, mental retardation, midface hypoplasia, and elliptocytosis: a new $\mathrm{X}$ linked contiguous gene deletion syndrome? J Med Genet 1998;35:273-8.

2 Barker DF, Hostikka SL, Zhou J, Chow LT, Oliphant AR, Gerken SC, Gregory MC, Skolnick MH, Atkin CL, Tryggvason K. Identification of mutations in the COL4A5 collagen gene in Alport syndrome. Science 1990;248:1224-7.

3 Piper MC, Pinnell LE, Darrah J, Maguire T, Byrne PJ. Construction and validation of the Alberta Infant Motor Scale (AIMS). Can J Public Health 1992;83(Suppl 2): S46-50.

4 Hoopes RR, Shrimpton AE, Knohl SJ, Hueber P, Hoppe B, Matyus J, Simckes A, Tasic V, Toenshoff B, Suchy SF. Dent disease with mutations in OCRL1. Am J Hum Genet 2005:76:260-7.

5 Andreoletti G, Ashton JJ, Coelho T, Willis C, Haggarty R, Gibson J, Holloway J, Batra A, Afzal NA, Beattie RM. Exome analysis of patients with concurrent pediatric inflammatory bowel disease and autoimmune disease. Inflamm Bowel Dis 2015;21:1229-36.

6 Christodoulou K, Wiskin AE, Gibson J, Tapper W, Willis C, Afzal NA, Upstill-Goddard R, Holloway JW, Simpson MA, Beattie RM. Next generation exome sequencing of paediatric inflammatory bowel disease patients identifies rare and novel variants in candidate genes. Gut 2013;62:977-84.

7 Li H, Handsaker B, Wysoker A, Fennell T, Ruan J, Homer N, Marth G, Abecasis G, Durbin R. The sequence alignment/map format and SAMtools. Bioinformatics 2009;25:2078-9.

8 Wang K, Li M, Hakonarson H. ANNOVAR: functional annotation of genetic variants from high-throughput sequencing data. Nucleic Acids Res 2010;38: e164-64.

9 Sherry ST, Ward MH, Kholodov M, Baker J, Phan L, Smigielski EM, Sirotkin K. dbSNP: the NCBI database of genetic variation. Nucleic Acids Res 2001;29:308-11.

10 Abecasis GR, Auton A, Brooks LD, 1000 Genomes Project Consortium. An integrated map of genetic variation from 1,092 human genomes. Nature 2012:491:56-65.

11 Exome Variant Server NHLBI. Exome Sequencing Project (ESP). Secondary Exome Sequencing Project (ESP), 2012. http://evs.gs. washington.edu/EVS/

12 Drmanac R, Sparks AB, Callow MJ, Halpern AL, Burns NL, Kermani BG, Carnevali P, Nazarenko I, Nilsen GB, Yeung G. Human genome sequencing using unchained base reads on self-assembling DNA nanoarrays. Science 2010;327:78-81.

13 Seaby EG, Pengelly RJ, Ennis S. Exome sequencing explained: a practical guide to its clinical application. Brief Funct Genomics 2016;15:374-84.

14 Jun G, Flickinger M, Hetrick KN, Romm JM, Doheny KF, Abecasis GR, Boehnke M, Kang HM. Detecting and estimating contamination of human DNA samples in sequencing and array-based genotype data. Am J Hum Genet 2012;91:839-48.

15 Pengelly RJ, Gibson J, Andreoletti G, Collins A, Mattocks CJ, Ennis S. A SNP profiling panel for sample tracking in whole-exome sequencing studies. Genome Med 2013:5:89.

16 Plagnol V, Curtis J, Epstein M, Mok KY, Stebbings E, Grigoriadou S, Wood NW, Hambleton S, Burns SO, Thrasher AJ. A robust model for read count data in exome sequencing experiments and implications for copy number variant calling. Bioinformatics 2012;28:2747-54

17 Mant A, Williams S, Roncoroni L, Lowry E, Johnson D, O'Kelly I. $\mathrm{N}$-Glycosylation-dependent Control of Functional Expression of Background Potassium Channels K2P3.1 and K2P9.1. J Biol Chem 2013;288:3251-64. (5.

18 Pollard KS, Hubisz MJ, Rosenbloom KR, Siepel A. Detection of nonneutral substitution rates on mammalian phylogenies. Genome Res 2010;20:110-21.

19 Kircher M, Witten DM, Jain P, O'Roak BJ, Cooper GM, Shendure J. A general framework for estimating the relative pathogenicity of human genetic variants. Nat Genet 2014;46:310.

20 Adzhubei IA, Schmidt S, Peshkin L, Ramensky VE, Gerasimova A, Bork P, Kondrashov AS, Sunyaev SR. A method and server for predicting damaging missense mutations. Nat Methods 2010;7:248-9.

21 Meloni I, Vitelli F, Pucci L, Lowry R, Tonlorenzi R, Rossi E, Ventura M, Rizzoni G, Kashtan C, Pober B. Alport syndrome and mental retardation: clinical and genetic dissection of the contiguous gene deletion syndrome in Xq22. 3 (ATS-MR). J Med Genet 2002;39:359-65.

22 Rodriquez JD, Bhat SS, Meloni I, Ladd S, Leslie ND, Doyne EO, Renieri A, DuPont $B R$, Stevenson RE, Schwartz CE. Intellectual disability, midface hypoplasia, facial hypotonia, and Alport syndrome are associated with a deletion in Xq22.3. Am J Med Genet A 2010;152:713-17.

23 von Mikecz A. The nuclear ubiquitin-proteasome system. J Cell Sc 2006;119:1977-84.

24 Lecker SH, Goldberg AL, Mitch WE. Protein degradation by the ubiquitin-proteasome pathway in normal and disease states. J Am Soc Nephrol 2006;17:1807-19.

25 Baldin V, Militello M, Thomas Y, Doucet C, Fic W, Boireau S, Jariel-Encontre I, Piechaczyk M, Bertrand E, Tazi J, Coux O. A novel role for PA28gamma-proteasome in nuclear speckle organization and SR protein trafficking. Mol Biol Cell 2008;19:1706-16

26 Gazou A, Riess A, Grasshoff U, Schäferhoff K, Bonin M, Jauch A, Riess O, Tzschach A. Xq22. 3-q23 deletion including ACSL4 in a patient with intellectual disability. Am J Med Genet A 2013;161:860-4.

27 Meloni I, Muscettola M, Raynaud M, Longo I, Bruttini M, Moizard M-P, Gomot M, Chelly J, des Portes V, Fryns J-P. FACL4, encoding fatty acid-CoA ligase 4 , is mutated in nonspecific X-linked mental retardation. Nat Genet 2002:30:436-40.

28 Robson WLM, Lowry RB, Leung AK. X-linked recessive nephritis with mental retardation, sensorineural hearing loss, and macrocephaly. Clin Genet 1994;45:314-17.

29 von Mering C, Jensen LJ, Snel B, Hooper SD, Krupp M, Foglierini M, Jouffre N, Huynen MA, Bork P. STRING: known and predicted protein-protein associations, integrated and transferred across organisms. Nucleic Acids Res 2005;33(suppl 1): D433-37.

30 Hoischen A, Landwehr C, Kabisch S, Ding X-Q, Trost D, Stropahl G, Wigger M, Radlwimmer B, Weber RG, Haffner D. Array-CGH in unclear syndromic nephropathies identifies a microdeletion in Xq22. 3-q23. Pediatr Nephrol 2009;24:1673-81.

31 Szklarczyk D, Franceschini A, Wyder S, Forslund K, Heller D, Huerta-Cepas J, Simonovic M, Roth A, Santos A, Tsafou KP. STRING v10: protein-protein interaction networks, integrated over the tree of life. Nucleic Acids Res 2015;43(Database issue):D447-52 